\title{
Inclusión de forrajes con distinta relación de fibra soluble e insoluble en la dieta de cerdos
}

\author{
González, A. ${ }^{\circledR}$; Figueroa, V.; Batista, C.; Casal, A.; Álvarez, A.; Saadoun A. y Astigarraga, L.
}

Departamento de Producción Animal y Pasturas. Facultad de Agronomía. Universidad de la República. Montevideo. Uruguay.

\section{PalABRAs Clave}

Alimentos alternativos.

Consumo.

Eficiencia.

Fibra dietaria.

\section{RESUMEN}

Se evaluó la inclusión progresiva de dos forrajes con diferente contenido en fibra soluble (FS), en dietas de cerdos desde la recría a la terminación. Los tratamientos fueron: dieta base testigo (TO) formulada en base a maíz y harina de soja; dieta base con alfalfa (T1) (Medicago sativa var. Chaná) o con achicoria (T2) (Cichorium intibus var. Lacerta) para las fases (F) de recría I (FI, 40-60 kg de PV), recría II (F2, 60-80 kg de PV) y terminación (F3, 80 a $100 \mathrm{~kg}$ de PV). Se utilizaron 27 animales con un peso inicial de $42 \mathrm{~kg}( \pm 2.3)$, en un diseño de parcelas al azar en tres tratamientos con nueve repeticiones. El peso final y la ganancia diaria no difirieron entre tratamientos. El consumo de Fibra Dietaria fue mayor en los tratamientos con forraje $(\mathrm{P}<0.05)$, mientras el consumo de FS fue mayor en la dieta con achicoria que en la dieta con alfalfa $(P<0.05)$. Este mayor consumo de Fibra Dietaria estuvo asociado a un mayor peso de los componentes del tracto gastrointestinal de los cerdos, en particular el colon en T2 ( $\mathrm{P}<0.05)$. Como consecuencia, T2 presentó el menor rendimiento de carcasa $(77.4 \%, P<0.05)$, respecto a TO y $\mathrm{Tl}$, los que fueron similares entre si $(80.0$ y $78.9 \%$ respectivamente, $\mathrm{P}=0.303)$. La inclusión progresiva de forraje posibilitó una adaptación física del tracto, mediante el aumento del estómago y colón, pero dicha adaptación afectó el rendimiento de carcasa.

\section{Different ratio of soluble and insoluble fiber in the pig diet}

\section{SUMMARY}

\section{ADDITIONAL KEYWORDS}

Alternative foods.

Dietary fiber.

Efficiency.

Feed intake.

\section{INFORMATION}

Cronología del artículo.

Recibido/Received: 20.02.2020

Aceptado/Accepted: 03.06.2020

On-line: 15.10 .2020

Correspondencia a los autores/Contact e-mail:

agonzalez@fagro.edu.uy

\section{INTRODUCCIÓN}

El uso de alimentos fibrosos ha adquirido un nuevo impulso, en respuesta a los beneficios que genera en la salud intestinal (Gong \& Yang 2012, Lindberg 2014) y el bienestar de los animales (Presto et al. 2019). Sin embargo, ha quedado constatado que la fracción fibrosa en la dieta de los cerdos reduce la utilización de los nutrientes y de la energía (Le Goff et al. 2002, Noblet \& Le Goff 2001), lo cual disminuye la ganancia diaria y el rendimiento de carcasa (Edwards 2003, Hansen et al. 2006). Pareciera que el aumento de la ingesta voluntaria de los animales que reciben forrajes en sus dietas es insuficiente para compensar la reducción en la concentración energética, y afectan así los indicadores productivos (Wüstholz et al. 2017). No obstante, las respuestas productivas podrían variar con el tipo de fibra presente, teniendo en cuenta que sus componentes solubles e insolubles alteran de forma diferente el desarrollo del tracto gastrointestinal (TGI), y por tanto 
la utilización de los nutrientes. Lyu et al. (2018) encontraron coeficientes de digestibilidad aparente más elevados en dietas con alimentos que contenían mayor concentración de fibra soluble (FS) respecto a la fibra insoluble (FI). Es posible que alimentos que muestren relaciones diferentes de FS/FI originen respuestas productivas diferentes. Adicionalmente, estudios sobre la inclusión de forraje en dieta de cerdos, reportan cambios en la composición lipídica de la carne, con incrementos en el contenido de ácido grasos poliinsaturados y mejorías en la relación de ácidos grasos (n6/n3), lo cual puede resultar beneficioso para la salud humana (Edwards 2005, Hansen et al. 2006). El objetivo de este experimento fue evaluar el efecto de la inclusión progresiva de dos forrajes con diferente tipo de fibra (FS/ FI) en una dieta convencional (maíz, harina de soja) ofrecida a cerdos en la etapa crecimiento y engorde sobre el consumo, rendimiento productivo y el perfil lipídico de la grasa intramuscular.

\section{MATERIAL Y MÉTODOS}

El experimento se desarrolló en la Estación de Pruebas Porcinas de la Facultad de Agronomía, Montevideo, Uruguay, de enero a abril de 2016. Los tratamientos evaluados fueron (Tabla I): dieta testigo T0 (maíz, harina de soja y núcleo vitamínico-mineral) formulada de acuerdo con las recomendaciones nutricionales del NRC (2012) para cerdos; dieta con inclusión de alfalfa (T1) con los mismos ingredientes de T0 y alfalfa (Medicago sativa var. Chaná); dieta con inclusión de achicoria (T3) con los mismos ingredientes de T0 y achicoria (Cichorium intibus var. Lacerta). Los forrajes fueron cortados en estado vegetativo, a una altura aproximada de $3 \mathrm{~cm}$ sobre el suelo, deshidratados y molidos (5 $\mathrm{mm}$ ) previo a ser incorporados como componentes de las raciones en niveles crecientes en función del peso vivo de los animales: 10\% de inclusión en el rango de 40-60 kg PV (Recría I o Fase 1, F1), 20\% de inclusión en el rango de $60-80 \mathrm{~kg}$ PV (Recría II o Fase 2, F2) y 30\% de inclusión en el rango de 80 a 100 kg PV (Terminación o Fase 3, F3) respectivamente (Tabla I).

Se utilizaron un total de 27 cerdos (hembras y machos castrados) de tres meses de edad con peso inicial $40 \pm 3.6 \mathrm{~kg}$ provenientes de un cruzamiento de hembra Landrace $x$ Large White con macho Híbrido terminal. Los animales fueron distribuidos en un diseño experimental de parcelas al azar con tres tratamientos y nueve repeticiones, siendo el animal la unidad experimental. Se alojaron en bretes individuales, con comedero tipo batea y bebedero automático tipo chupete. Durante el experimento, las temperaturas registradas oscilaron entre 21 y $27^{\circ} \mathrm{C}$. La cantidad diaria de ración a ofrecer se calculó en función a los requerimientos de Energía Metabolizable (EM) y Lisina digestible ileal aparente (LIDA) para mantenimiento y crecimiento de acuerdo con la ganancia diaria esperada de los animales (NRC 2012). El alimento se ofrecía a las 9:00 am y 3:00 pm, con la cantidad diaria dividida en partes iguales entre las dos comidas. Se tomaron muestras del ofrecido diariamente y así formar una muestra compuesta por tratamiento y por fase. El rechazo se retiraba diariamente a primera hora de la mañana, se identificaba por fecha y animal. Todas las muestras fueron conservadas a $-20{ }^{\circ} \mathrm{C}$ para su posterior análisis químico. Los animales se pesaron semanalmente hasta el peso de faena, momento en que fueron enviados a un matadero comercial. Posterior a la faena se registró el peso de los componentes vacíos del tracto gastrointestinal: estómago (E), intestino delgado (ID), colon (CO) ciego (CI) y recto (RE). Pasadas las 24 horas del sacrificio, se registró el peso de carcasa con cabeza y se extrajo a la altura de la 12a costilla del lado izquierdo una muestra de $200 \pm 20 \mathrm{~g}$ del musculo Longissimus thoracis, de cada animal. En ese punto se midió el espesor de grasa dorsal (EGD, mm). Las muestras de carne se colocaron en bolsas de plástico individuales y se envasaron al vacío a $-20{ }^{\circ} \mathrm{C}$ para su posterior determinación de los ácidos grasos (AG, \%). Las determinaciones de composición química de los ingredientes y de las dietas ofrecidas fueron realizadas en el Laboratorio de Nutrición Animal de la Facultad de Agronomía. Se determinó Materia seca (MS) (AOAC 2012, Ref.: 934.01), Cenizas (C), Nitrógeno (N), Fibra Detergente Neutro (FDN), Fibra Detergente Ácida (FDA) y Lignina, de acuerdo con la metodología descrita en AOAC (2012, 942.05, 954.01; 2002.04; 973.18 respectivamente). Se utilizó el método enzimático-gravimétrico para la determinación de Fibra dietaria (FD), soluble (FS) e insoluble (FI) de los ingredientes (AOAC 2012; 985.29, 993.19, 991.42). Para cuantificar el contenido de grasa intramuscular se realizó una extracción de los lípidos totales con la técnica de Folch, Lees and Stanley (1957) basada en el uso de una mezcla de cloroformo y metanol. Se realizó una metilación en frío de los ácidos grasos extraídos que se cuantificaron con una columna capilar CPSIL-88 de $100 \mathrm{~m}$ de longitud, acoplada a un cromatógrafo Perkin Elmer Split/Splitless ClARUs 500. Las rampas de temperatura, presión de hidrógeno y aire se describen en Terevinto, Cabrera \& Saadoum (2019). La ganancia diaria (GD, kg/día) se calculó con el peso inicial, el peso final y la duración en días de cada Fase. Se calculó la eficiencia a partir del índice de conversión del alimento (CA, $\mathrm{kg} \mathrm{MO} / \mathrm{kg}$ ) determinado como la cantidad de $\mathrm{MO}$ consumida para ganar $1 \mathrm{~kg}$ de PV. Se calculó el rendimiento $(R, \%)$ como el cociente entre el peso de carcasa con cabeza y el peso vivo en el matadero. El peso de las vísceras fue expresado como porcentaje del peso vivo a la faena. Las variables consumo, peso vivo, ganancia de peso y conversión alimentaria, fueron analizadas utilizando PROC MIXED del paquete estadístico SAS (versión 9.1.3, 2006). El modelo incluyó el efecto Tratamiento, Fase, y sus interacciones, y el peso al inicio del experimento como covariable. Las variables peso post mortem, rendimiento, peso de vísceras, espesor de grasa dorsal y composición de ácidos grasos fueron analizadas con PROC MIXED, con efecto fijo Tratamiento. El animal se consideró como efecto aleatorio. Todas las medias fueron comparadas por test de Tukey. Las diferencias se consideraron significativas a nivel de $\mathrm{P} \leq 0.05$. Los procedimientos experimentales y las condiciones del cuidado de los animales fueron aprobados por el Comité de Ética de Experimentación Animal de la Universidad de la República, Uruguay (Exp. No 021130-001011-16).

\section{RESULTADOS}


La composición química de los forrajes utilizados en este experimento se presenta en la Tabla II. Los datos se expresan como porcentaje de la $\mathrm{MO}$, debido

Tabla I. Ingredientes y composición química calculada de las dietas experimentales (\%, en base al alimento) (Ingredients and calculated chemical composition of the experimental diets (as-fed basis)).

\begin{tabular}{|c|c|c|c|c|c|c|c|c|c|}
\hline \multirow[b]{2}{*}{ Ingredientes } & \multicolumn{3}{|c|}{ Fase 1} & \multicolumn{3}{|c|}{ Fase 2} & \multicolumn{3}{|c|}{ Fase 3} \\
\hline & T0 & T1 & $\mathrm{T} 2$ & TO & T1 & $\mathrm{T} 2$ & T0 & T1 & $\mathrm{T} 2$ \\
\hline Maíz & 76.9 & 67.9 & 67.3 & 84.8 & 65.3 & 64 & 88 & 62.8 & 60.8 \\
\hline Harina Soja & 21.2 & 20.1 & 20.8 & 13.6 & 13.4 & 14.8 & 11 & 5.9 & 7.9 \\
\hline Núcleo ${ }^{(1)}$ & 2.0 & 2.0 & 2.0 & 1.6 & 1.3 & 1.3 & 1.0 & 1.3 & 1.3 \\
\hline Forraje deshidratado & 0 & 10 & 10 & 0 & 20 & 20 & 0 & 30 & 30 \\
\hline \multicolumn{10}{|l|}{$\begin{array}{l}\text { Composición química } \\
\text { calculada }\end{array}$} \\
\hline $\mathrm{PC}, \%$ & 15.3 & 15.7 & 15.6 & 12.4 & 15.4 & 14.5 & 11.3 & 13.6 & 12.5 \\
\hline LIDA, \% & 0.9 & 0.9 & 0.9 & 0.7 & 0.7 & 0.7 & 0.6 & 0.5 & 0.5 \\
\hline EM, Mcal/kg & 3.3 & 3.1 & 3.1 & 3.3 & 3.0 & 2.9 & 3.3 & 2.8 & 2.6 \\
\hline
\end{tabular}

(1) Núcleo vitamínico-mineral: Humedad máx.10\%; Minerales totales: 80\%; Calcio: 16-21,5\%; Fósforo: 6,5-8,5\%; Solubilidad de P en ácido cítrico: 90\%; Sodio: 6\%; Lisina: 0,5\%; Cenizas insolubles: 8\%; Vitaminas. T0: Dieta testigo; T1: Dieta con alfalfa; T2:

Dieta con achicoria; PC: Proteína cruda; LIDA: Lisina Digestible ileal aparente; EM: Energía Metabolizable.

al elevado contenido de cenizas que presentó el forraje de achicoria (30\%, base seca). La achicoria y la alfalfa mostraron similares contenidos de MS, MO, PC, Lignina y de Fibra tanto FDN como FD. Sin embargo, la composición de la FD difirió entre forrajes, con valores de FI superiores para la alfalfa $(\mathrm{P}=0.014)$, mientras que la concentración de FS de la achicoria fue casi tres veces mayor a la concentración de FS de la alfalfa $(\mathrm{P}<0.001)$.

Los pesos iniciales y finales de los animales no mostraron diferencias entre tratamientos $(\mathrm{P}>0.05)$, pero difirieron entre Fases $(\mathrm{P}<0.001)$ (Tabla III). La duración en días de cada Fase también fue similar entre

Tabla II. Composición química de los forrajes (Chemical composition of forages).

\begin{tabular}{lccc}
\hline Fracciones & Alfalfa & Achicoria & $P$ \\
MS, g/kg Alimento & $877 \pm 5$ & $882 \pm 8$ & 0.376 \\
MO, g/kg MS & $823 \pm 68$ & $693 \pm 68$ & 0.080 \\
$\mathrm{PC}, \mathrm{g} / \mathrm{kg} \mathrm{MO}$ & $239 \pm 35$ & $208 \pm 23$ & 0.269 \\
$\mathrm{FDN}, \mathrm{g} / \mathrm{kg} \mathrm{MO}$ & $517 \pm 49$ & $438 \pm 43$ & 0.101 \\
$\mathrm{FDA}, \mathrm{g} / \mathrm{kg} \mathrm{MO}$ & $312 \pm 25$ & $281 \pm 27$ & 0.217 \\
$\mathrm{Lig} ., \mathrm{g} / \mathrm{kg} \mathrm{MO}$ & $120 \pm 7$ & $116 \pm 13$ & 0.669 \\
$\mathrm{FD}, \mathrm{g} / \mathrm{kg} \mathrm{MO}$ & $519 \pm 29$ & $527 \pm 12$ & 0.690 \\
$\mathrm{FI}, \mathrm{g} / \mathrm{kg} \mathrm{MO}$ & $468^{\mathrm{a}} \pm 27$ & $390^{\mathrm{b}} \pm 17$ & 0.014 \\
$\mathrm{FS}, \mathrm{g} / \mathrm{kg} \mathrm{MO}$ & $51^{\mathrm{b}} \pm 8$ & $136^{\mathrm{a}} \pm 11$ & $<0.001$ \\
$\mathrm{FS} / \mathrm{Fl}, \%$ & $11^{\mathrm{b}} \pm 1$ & $35^{\mathrm{a}} \pm 2$ & $<0.001$ \\
\hline
\end{tabular}

a,b: medias de tratamientos con diferentes letras, difieren significativamente $(\mathrm{P}<0.05)$. MS: Materia seca; MO: Materia orgánica; PC: Proteína Cruda; FDN: Fibra Detergente Neutro; FDA: Fibra Detergente Acida; Lig.: Lignina; FD: Fibra Dietaria; FI: Fibra Insoluble; FS: Fibra Soluble tratamientos (22 días en promedio), por lo cual las ganancias diarias no mostraron diferencias entre tratamientos ( $\mathrm{P}=0.129)$, aunque sí entre Fases $(880,903,821$ g/d para la Fase 1, Fase 2 y Fase 3 respectivamente, $\mathrm{P}=0.048$ ).

El consumo de MO fue similar entre tratamientos en la Fase 1 y en la Fase 2, pero en la Fase 3 el consumo de $\mathrm{MO}$ en las dietas con inclusión de forraje fue mayor con respecto a la dieta testigo $(\mathrm{P}<0.027)$. El consumo de $\mathrm{PC}$ fue mayor $(\mathrm{P}<0.001)$ para los tratamientos con forraje en todas las Fases (Tabla IV).

El consumo de FDN fue diferente entre tratamientos $(\mathrm{P}<0.001)$. Los animales que recibieron dietas con forraje consumieron diariamente mayor cantidad de FDN, el cual representó el 1\% del PV en comparación al 0,8\% del PV para la dieta testigo en cada fase de evaluación. Los consumos de FD y de FI fueron similares entre tratamientos en Fase 1, sin embargo, en la Fase 2 y la Fase 3 los animales recibiendo dietas con forrajes presentaron mayores consumos que el testigo $(\mathrm{P}<0.001)$. Para FS, los animales que recibieron T2 registraron en todas las fases, consumos diarios superiores respecto a T0 y T1 $(\mathrm{P}<0.001)$. La eficiencia calculada a partir de la cantidad de $\mathrm{MO}$ consumida por unidad de peso ganado fue similar $(\mathrm{P}=0.454)$ entre tratamientos con forrajes e inferior a la dieta Testigo (2.5 vs. $2.8 \mathrm{~kg} \mathrm{MO} / \mathrm{kg}$, para dietas sin y con forrajes respectivamente, $\mathrm{P}<0.026$ )

Los cerdos del tratamiento $\mathrm{T} 0$ presentaron mayor peso de carcasa que los que recibieron dietas con forrajes $(\mathrm{P}<0.001)$, mientras que el $\mathrm{R}(\%)$ fue similar entre los tratamientos $\mathrm{T} 0$ y T1, pero menor para el tratamiento $\mathrm{T} 2$ con respecto al tratamiento T0 $(\mathrm{P}=0.019)$. (Tabla V). En cuanto al peso relativo de vísceras a la faena en cada tratamiento, los animales que recibieron dietas con forrajes mostraron valores relativos mayores de peso de estómago ( $+15 \%, \mathrm{P}<0.001)$, de peso de intestino del- 
Tabla III. Indicadores de performance para cada tratamiento y fase de crecimiento y engorde de cerdos (Indicators of performance for each treatment and phase of growing and finish pig).

\begin{tabular}{lcccccccccccc}
\hline & \multicolumn{3}{c}{ Fase 1 } & \multicolumn{3}{c}{ Fase 2 } & \multicolumn{3}{c}{ Fase 3 } & & P \\
Ítem & T0 & T1 & T2 & T0 & T1 & T2 & T0 & T1 & T2 & Trat. & Fase & Trat x Fase \\
PV inicial, kg & 41.8 & 43.2 & 42.6 & 62.6 & 62.5 & 61.2 & 80.8 & 80.2 & 81.0 & 0.868 & $<0.001$ & 0.481 \\
PV final, kg & 62.6 & 62.5 & 61.2 & 83.1 & 80.2 & 82.3 & 100.1 & 96.0 & 98.4 & 0.062 & $<0.001$ & 0.392 \\
Días & 22 & 22 & 22 & 21 & 20 & 23 & 20 & 22 & 21 & 0.286 & 0.183 & 0.205 \\
Ganancia, g/d & 913 & 846 & 881 & 915 & 891 & 905 & 870 & 753 & 841 & 0.129 & 0.048 & 0.840 \\
\hline
\end{tabular}

T0: Dieta testigo; T1: Dieta con alfalfa; T2: dieta con achicoria.

$\mathrm{a}, \mathrm{b}$ : medias de tratamientos con diferentes letras dentro de fase, difieren significativamente $(\mathrm{P}<0.05)$. PV: peso vivo.

\begin{tabular}{|c|c|c|c|c|c|c|c|c|c|c|c|c|}
\hline \multirow{2}{*}{$\begin{array}{l}\text { Ítem } \\
\text { Consumo } \\
\text { diario }\end{array}$} & \multicolumn{3}{|c|}{ Fase 1} & \multicolumn{3}{|c|}{ Fase 2} & \multicolumn{3}{|c|}{ Fase 3} & \multicolumn{3}{|c|}{$\mathrm{P}$} \\
\hline & T0 & $\mathrm{T} 1$ & T2 & T0 & $\mathrm{T} 1$ & $\mathrm{~T} 2$ & T0 & T1 & $\mathrm{T} 2$ & Trat & Fase & Tratx Fase \\
\hline $\mathrm{MS}, \mathrm{kg} / \mathrm{d}$ & 2.0 & 2.1 & 2.1 & $2.4^{b}$ & $2.5^{\mathrm{ab}}$ & $2.7^{\mathrm{a}}$ & $2.7^{\mathrm{b}}$ & $2.9^{b}$ & $3.1^{\mathrm{a}}$ & $<0.001$ & $<0.001$ & 0.023 \\
\hline $\mathrm{MO}, \mathrm{kg} / \mathrm{d}$ & 1.8 & 1.9 & 1.9 & 2.3 & 2.4 & 2.4 & $2.5^{\mathrm{b}}$ & $2.7^{a}$ & $2.8^{a}$ & $<0.001$ & $<0.001$ & 0.137 \\
\hline$P C, g / d$ & $329^{b}$ & $387^{a}$ & $392^{a}$ & $327^{\circ}$ & $406^{b}$ & $448^{a}$ & $347^{b}$ & $427^{a}$ & $403^{a}$ & $<0.001$ & $<0.001$ & $<0.001$ \\
\hline FDN, g/d & $450^{\mathrm{b}}$ & $555^{a}$ & $541^{a}$ & $606^{b}$ & $747^{a}$ & $693^{a}$ & $757^{\mathrm{b}}$ & $1000^{a}$ & $956^{a}$ & $<0.001$ & $<0.001$ & $<0.001$ \\
\hline FD, g/d & 585 & 633 & 637 & $725^{b}$ & $828^{a}$ & $844^{a}$ & $900^{\mathrm{b}}$ & $1051^{a}$ & $1127^{a}$ & $<0.001$ & $<0.001$ & $<0.001$ \\
\hline $\mathrm{Fl}, \mathrm{g} / \mathrm{d}$ & 550 & 591 & 585 & $683^{b}$ & $773^{a}$ & $756^{a}$ & $850^{\mathrm{b}}$ & $970^{\mathrm{a}}$ & $976^{a}$ & $<0.001$ & $<0.001$ & 0.004 \\
\hline FS, g/d & $36^{b}$ & $42^{b}$ & $52^{a}$ & $42^{c}$ & $55^{b}$ & $89^{a}$ & $50^{c}$ & $81^{b}$ & $151^{a}$ & $<0.001$ & $<0.001$ & $<0.001$ \\
\hline $\begin{array}{l}\mathrm{CA}, \mathrm{kg} \mathrm{MO} / \\
\mathrm{kg}\end{array}$ & 2.1 & 2.2 & 2.2 & 2.4 & 2.5 & 2.8 & $2.9^{b}$ & $3.7^{\mathrm{a}}$ & $3.4^{\mathrm{ab}}$ & 0.026 & $<0.0001$ & 0.1202 \\
\hline
\end{tabular}

a,b,c: medias de tratamientos con diferentes letras dentro del periodo, difieren significativamente $(P<0.05)$.

T0: Dieta testigo; T1: Dieta con alfalfa; T2: dieta con achicoria MS: Materia seca; MO: Materia orgánica; PC: Proteína cruda; EM: Energía Metabolizable; FDN: Fibra Detergente Neutro; FD: Fibra Dietaria, FI: Fibra insoluble; FS: Fibra soluble; CA: Conversión alimentaria.

gado $(+11 \%, \mathrm{P}<0.001)$ con respecto a los animales del tratamiento T0. El peso relativo del colon fue mayor en los animales del tratamiento T2 (1,81\% del PV), con respecto a los animales de los tratamientos T1 y T0 que no difirieron entre sí (1.52\% del PV en promedio, $\mathrm{p}<0.001)$. El peso relativo de ciego $(0.22 \%$ del PV) y recto $(0.23 \%$ del PV) no difirió entre tratamientos (Tabla V).
No se observó diferencias en el espesor de grasa dorsal (mm), en el perfil de ácidos grasos, en la relación AG ins/AG sat y tampoco en la composición relativa entre AG n6/n3 entre tratamientos (Tabla VI).

\section{DISCUSIÓN}

\begin{tabular}{lcccc}
\hline Tabla V. Efecto del tratamiento sobre el peso de la canal, el rendimiento y los componentes del tracto gastro- \\
intestinal (como proporción del peso vivo) para los diferentes tratamientos (Effect of treatment on channel weight, \\
performance and components of the gastrointestinal tract (as a proportion of body weight) for different treatments). \\
\hline Ítem & T0 & T1 & T2 & P \\
Peso 24h post mortem, kg & $81.6^{\mathrm{a}}$ & $76.5^{\mathrm{b}}$ & $76.2^{\mathrm{b}}$ & $<0.001$ \\
Rendimiento, \% & $80.0^{\mathrm{a}}$ & $78.9^{\mathrm{ab}}$ & $77.4^{\mathrm{b}}$ & 0.019 \\
Peso de las vísceras (expresado como \% del PV) & & & & \\
Estómago & $0.64^{\mathrm{b}}$ & $0.75^{\mathrm{a}}$ & $0.76^{\mathrm{a}}$ & $<0.001$ \\
Intestino delgado & $4.12^{\mathrm{b}}$ & $4.46^{\mathrm{a}}$ & $4.77^{\mathrm{a}}$ & $<0.001$ \\
Colon & $1.60^{\mathrm{b}}$ & $1.45^{\mathrm{b}}$ & $1.81^{\mathrm{a}}$ & $<0.001$ \\
Ciego & 0.22 & 0.22 & 0.23 & 0.613 \\
Recto & 0.21 & 0.23 & 0.24 & 0.317
\end{tabular}

T0: Dieta testigo; T1: Dieta con alfalfa; T2: dieta con achicoria a,b: medias de tratamientos con diferentes letras, difieren significativamente $(\mathrm{P}<0.05)$. PV: peso vivo. 
Tabla VI. Características de la canal y perfil de ácidos grasos (Characteristics of the carcass and fatty acid profile).

\begin{tabular}{lcccc}
\hline Item & T0 & T1 & T2 & P \\
EGD, mm & 19.38 & 17.78 & 20.89 & 0.083 \\
Lípidos totales, \% & 2.37 & 2.72 & 2.89 & 0.764 \\
AG Saturados, \% & 43.8 & 42.8 & 44.1 & 0.475 \\
AG Insaturados, \% & 55.3 & 56.1 & 54.9 & 0.570 \\
AG ins/AG sat, & 1.27 & 1.31 & 1.25 & 0.533 \\
AG n3, \% & 0.787 & 0.823 & 0.937 & 0.396 \\
AG n6, \% & 4.86 & 5.46 & 5.29 & 0.396 \\
AG n6/n3 & 6.35 & 6.70 & 5.89 & 0.413 \\
\hline
\end{tabular}

T0: Dieta testigo; T1 Dieta con alfalfa; T2: Dieta con achicoria nivel de diferencia significativa $(P<0.05)$. EGD: Espesor de Grasa Dorsal, AG: Ácidos Grasos.

La composición química no varió significativamente entre ambos forrajes, si ésta es expresada en función del contenido de MO. De hecho, el alto contenido de cenizas en las muestras analizadas de achicoria podría estar asociado a una mayor contaminación con tierra por su porte tipo roseta a nivel del suelo. El contenido de FD fue similar entre forrajes $(429 \mathrm{~g} / \mathrm{kg}$ MO en promedio), pero la proporción FS/FI fue tres veces mayor en el caso de la achicoria que en el caso de la alfalfa (Tabla II). En términos generales, los valores de composición química registrados tanto para alfalfa como para achicoria concuerdan con los reportados en la bibliografía (Moreno \& Rosas 2012, Muniz \& Silva 2014). La alfalfa ha sido uno de los forrajes más utilizados en las dietas de los cerdos, por su aporte en proteínas y minerales, a pesar de que su inclusión en las dietas puede disminuir la digestibilidad de los nutrientes por la presencia de componentes indigestibles de la pared celular (Chen et al. 2015). Los valores de FDN y FD son prácticamente similares en la alfalfa (517 y $519 \mathrm{~g} / \mathrm{kg}$ MO respectivamente). La FD de la alfalfa está presente principalmente como celulosa y hemicelulosa, componentes de la fibra insoluble. Guillon et al (2007) reportan que un $94 \%$ de la fibra dietética de la alfalfa es fibra insoluble, similar a los valores hallados en este trabajo ( $90 \%$ FI/FD). La celulosa y la hemicelulosa pueden ser relativamente resistentes a la fermentación microbiana en el intestino grueso de los cerdos, dependiendo de la composición de la matriz que embebe la celulosa que la vuelve menos disponible al ataque microbiano con el avance de la madurez del órgano vegetal en el momento de la cosecha (Bach Knudsen 2001). En este trabajo, dado el estado vegetativo al corte (30 días de crecimiento), es dable esperar que la misma presentara un mayor grado de aprovechamiento en el tracto gastrointestinal de los animales.

La achicoria ha sido un forraje utilizado frecuentemente en los sistemas de producción bovina, con escasos reportes de su uso en la alimentación de cerdos.
Estudios de composición realizados por Sun et al (2006) describen que las paredes de las hojas de achicoria no están lignificadas y en ellas predominan los polisacáridos pépticos, que son altamente degradados por microorganismos. En este trabajo, el contenido de FDN presentó una tendencia a ser menor en la achicoria con respecto a la alfalfa (438 vs. $517 \mathrm{~g} / \mathrm{kg}$ MO para achicoria y alfalfa respectivamente, $\mathrm{P}=0.101)$. Sin embargo, la achicoria presentó un contenido de FD similar a la alfalfa (517 y $527 \mathrm{~g} / \mathrm{kg}$ MO respectivamente, $\mathrm{P}=0.690$ ). El contenido de FD mayor a FDN que presenta la achicoria es atribuido al contenido de sustancias pécticas presente en la laminilla media de la pared celular, que se caracterizan por su alta solubilidad (Barry 1998). De hecho, la FS de la achicoria fue 2.5 veces mayor al contenido de FS de alfalfa ( $136 \mathrm{~g} / \mathrm{kg}$ MO vs. $51 \mathrm{~g} / \mathrm{kg}$ MO para achicoria y alfalfa respectivamente, $\mathrm{P}<0.001)$. Las pectinas formadas por un grupo heterogéneo de polisacáridos, homogalacturonanos, ramnogalacturonanos, están unidas por enlaces tipo $\alpha$ como los enlaces presentes en el almidón (Bach \& Calsamiglia 2006) y su alto contenido de ácido urónico (80-90g/ kg de MS) las hace fermentable para los cerdos. (Ivarsson et al. 2010ba). Ivarsson (2010b) utilizando niveles de inclusión del 16\% de harina de achicoria en dietas ofrecidas a lechones en posdestete, no obtuvo diferencias en consumo, ganancia diaria y eficiencia de conversión respecto a dietas en base a cereales. En el mismo sentido, los indicadores productivos presentados en la Tabla III no dan cuenta de una diferencia en las ganancias de los cerdos según el tipo de forraje incluido en la dieta, y tampoco difieren de las ganancias de los cerdos alimentados con la dieta T0. El peso final y la ganancia diaria fueron similares entre tratamientos dentro de la misma Fase experimental. Los valores de ganancia diaria reportados para este experimento se encuentran dentro del rango esperado para cada etapa de crecimiento, en animales que reciben dietas concentradas (NRC 2012). Diferentes fueron los resultados reportados por Hansen et al. (2006) y Wüstholz et al. (2017), quienes obtuvieron ganancias menores a las esperadas al incluir forraje ensilado en sustitución del concentrado. Las menores ganancias diarias de los cerdos reportados por estos autores podrían estar asociada al contenido agua de los forrajes, que incrementa el efecto de distensión del tracto gastrointestinal de los animales y tiende a deprimir el consumo (Forbes 2009). En la Tabla IV se presenta el consumo de los animales según tratamiento dentro de cada Fase. Los rechazos promedio fueron 2,7 y $4 \%$ con respecto a la cantidad ofrecida, para las dietas T0, T1 y T2 respectivamente. El consumo de MO fue similar entre tratamientos en la Fase 1 y en la Fase 2, pero difirió en la Fase 3, siendo mayor en las dietas con inclusión de 30\% de forraje en comparación a la dieta T0 $(+10 \%)$. En cuanto al consumo de proteína cruda, éste siempre fue mayor en el caso de la dieta con inclusión de forrajes, asociado al buen contenido de proteína de estas especies forrajeras como ya fuera reportado para alfalfa (Chen et al. 2015) y achicoria (Barry 1998). Sin embargo, como era de esperar, las mayores diferencias entre tratamientos se observan a nivel del consumo de las fracciones Fibra. El consumo de fibra como FDN, siempre fue mayor $(+20 \%)$ en los tratamientos con inclusión de forraje en cualquiera de 
las tres Fases, mientras que el consumo de FD fue mayor a la dieta Testigo luego del $20 \%$ de inclusión de forraje, esto es, en las Fase 2 y 3 . Las recomendaciones nutricionales incluyen valores mínimos de $11 \%$ y máximos de $15 \%$ para FDN, en cerdos en crecimiento y terminación (NRC 2012, De Blas, Gasa \& Mateos 2013). En este trabajo, el consumo de fibra como FDN alcanzó valores de $36 \%$ en la dieta consumida por los cerdos (Fase 3), lo cual representó aprox. 1\% PV de FDN consumido. En cuanto al consumo diario de FD, no se encontraron referencias de recomendaciones, ni en cuanto al consumo de fibra soluble (FS) e insoluble (FI). El consumo de FI acompañó la misma tendencia observada en el consumo de FD, es decir fue mayor en las dietas con inclusión de forraje en las Fases 2 y 3 (+ 13\% en promedio), pero las mayores diferencias se registraron en el consumo de FS, en particular en el caso de la dieta T2, que presentó mayores consumos en las tres Fases con respecto a la dieta T0 $(+44 \%,+112 \%,+200 \%$ en Fase 1, Fase 2, y Fase 3 respectivamente). El mayor consumo de FS, podría estar explicado por la abundancia de pectinas de la achicoria (Barry 1998). Fleming and Lee (1983) reportan que la incorporación de pectinas, como fuente de FS, se asocia a una disminución de la ingesta de alimento como consecuencia de un aumento en el tiempo de tránsito a través del tracto gastrointestinal. No obstante, en este trabajo, el consumo de los animales que recibieron la dieta con inclusión de achicoria fue igual (Fase 1 y Fase 2 ) o mayor (+10\% en Fase 3) que el consumo de los animales alimentados con la dieta T0. Es posible que la inclusión progresiva de forraje, en este caso de achicoria, en la dieta de los animales desde la recría I a la terminación acompañando así el crecimiento del animal, haya posibilitado una adaptación física del tracto al mayor contenido de FS, favoreciendo la utilización de los nutrientes y permitiendo mantener las respuestas productivas observadas en la Tabla III. Según Ellis and Augspurger (2001), los cerdos en crecimiento modifican su consumo con el objetivo de mantener constante la ingesta diaria de energía, hasta que las señales inhibitorias a lo largo del tracto limitan el mismo. Sin embargo, la sensibilidad a las señales del tracto gastrointestinal en el control a corto plazo de la ingesta parecería depender del estado metabólico del cerdo y del tiempo de acostumbramiento a las dietas (Gregory 2002, McDonald et al. 2001). En este sentido, para las dietas de este trabajo, el incremento en el consumo estaría asociado al incremento de la ingesta de energía al disminuir la concentración energética de la misma por mayor nivel de inclusión de forraje. Esta compensación es posible dentro de determinados límites. Black et al. (1986) reportan como límite crítico inferior el valor de $2.35 \mathrm{Mcal}$ de ED/kg, lo equivale a $2.26 \mathrm{Mcal}$ de EM/ $\mathrm{kg}$, considerando una ineficiencia del 4\% (Noblet el al 1994). En este trabajo, la concentración energética de las dietas experimentales estuvo siempre por encima del límite crítico inferior reportado por estos autores (Tabla I). Los valores de energía, expresados como EM, presentaron un valor mínimo de 2.67 Mcal/kg para T2 en la Fase 3. Las restantes dietas presentaron valores superiores, por lo cual es posible pensar que los animales podían alcanzar sus requerimientos energéticos utilizando la estrategia de incrementar el consumo aun con niveles del
$30 \%$ de inclusión de forraje (Fase 3), sin que las señales de saciedad actuaran como factor limitante. Al analizar la Conversión Alimenticia, se observa una disminución de los valores desde la Fase 1 a la Fase 3, acompañando el crecimiento del animal. Este comportamiento es el esperado, acorde con la evolución en la deposición grasa: músculo de los animales (Gaines, Peterson \& Mendoza 2012). Por otra parte, esas disminuciones fueron mayores para las dietas con forraje y mayor nivel de inclusión, por ende, de menor densidad energética (Beaulieu, Wiliams, \& Patience 2009), pero dentro de los rangos obtenidos para dietas fibrosas (Wüstholz et al. 2017). El peso $24 \mathrm{~h}$ post mortem fue significativamente mayor en los cerdos alimentados con la dieta T0 en comparación a los animales alimentados con las dietas con inclusión de forraje (81.6 vs. $76.3 \mathrm{~kg}, \mathrm{P}<0.001)$. Esta diferencia está asociada al peso de las vísceras del tracto gastrointestinal. Las adaptaciones progresivas del tracto gastrointestinal que realizaron los animales a la mayor ingestión de fibra dietaria y de fibra soluble quedan de manifiesto en las diferencias encontradas en el peso vacío de los componentes del tracto (Tabla V). Los animales que recibieron dietas con mayor contenido de fibra presentaron mayor peso de estómago e intestino en comparación a los animales de la dieta Testigo. McDonald et al. (2001) observaron que la ingestión y la fermentación de la fibra dietética aumentan el tamaño y la longitud de los órganos digestivos, incluido el intestino delgado, el ciego y el colon de los cerdos. Sin embargo, en este trabajo, fue mayor el peso del colon en el caso de la dieta con mayor contenido de fibra soluble (T2). Resultados similares fueron obtenidos por Ivarsson et al. (2010b) con niveles de inclusión del 16\% en lechones, concluyendo que la achicoria estimula el desarrollo del colon. Finalmente, el rendimiento de carcasa fue menor para los animales alimentados con la dieta con inclusión de achicoria con respecto al rendimiento de los animales de la dieta T0 (77.4 vs. $80.0 \%, \mathrm{P}=0.019$ ). En cuanto al espesor de grasa dorsal no se observaron diferencias asociadas a la inclusión de forraje en la dieta (Tabla VI). El grado de engrasamiento de los animales de los tres tratamientos estuvo dentro de los valores esperados para el tipo genético utilizado (Viana et al. 2019, Wüstholz et al. 2017). No se observaron diferencias en el perfil de ácidos grasos (saturados, insaturados y poliinsaturados) de la grasa intramuscular en los animales que recibieron las dietas con inclusión de forraje. Es de destacar que las diferencias en el contenido de PUFA en la grasa intramuscular ha sido reportado en animales que recibieron forraje fresco (Nilzén et al. 2001), mientras que en este ensayo el forraje fue suministrado seco. Tomando en cuenta el precio de mercado local (Cámara Mercantil de Productos del País [CMPP] 2018), la inclusión de los forrajes en las dietas representó una reducción en el costo por kg de ración, del $4 \%$ para la F1 y del 13\% para F2 y F3 respecto a la dieta T0. Esta reducción de costos puede ser mayor si tomamos en cuenta que la producción de cerdos se encuentra integrada a un sistema de producción diversificado, donde los forrajes pueden ser producidos en el propio establecimiento.

\section{CONCLUSION}


Niveles crecientes de forraje en la dieta de cerdos desde la etapa de recría hasta la etapa de terminación permitieron obtener resultados productivos similares a los animales alimentados con una dieta convencional. La inclusión progresiva de forraje permitiría una adaptación física del tracto al mayor contenido de Fibra, pero se incrementaría el peso del tracto gastrointestinal reduciendo significativamente el rendimiento de carcasa. Sin embargo, el rendimiento de carcasa fue diferente entre los tratamientos con forraje, siendo menor en el tratamiento con inclusión de achicoria por mayor peso relativo de las vísceras (colon). Esta diferencia podría estar asociada a la distinta relación Fibra Soluble/Fibra Insoluble entre ambos forrajes. La mayor inclusión de forraje deshidratado en la dieta de los cerdos no afectó el espesor de la grasa subcutánea, ni el perfil lipídico de la grasa intramuscular. A futuro, incluir el estudio de los parámetros de salud intestinal y de bienestar animal según tipo de Fibra Soluble/Insoluble en la dieta, permitiría avanzar en el conocimiento de los efectos de la inclusión de forrajes en la dieta de cerdos en crecimiento y terminación.

\section{BIBLIOGRAFÍA}

AOAC 2012, Official Methods of Analysis of AOAC International, 19th (ed) AOAC International, Gaithersburg, MD, USA, Official Method.

Bach, A \& Calsamiglia, S 2006, 'La fibra en los rumiantes ¿Química - física?' FEDNA Madrid, España: FEDNA, pp. 99-113, http:// fundacionfedna.org/sites/default/files/06CAP VI.pdf.

Bach Knudsen, KE 2001, 'The nutritional significance of "dietary fibre" analysis', Animal Feed Science and Technology, vol. 90, no. 1, pp. 3-20.

Barry, TN 1998, 'The feeding value of chicory (Cichorium intybus) for ruminant livestock', The Journal of Agricultural Science, vol. 131, no. 3, pp. 251-257.

Beaulieu, AD, Williams, NH \& Patience, JF 2009, 'Response to dietary digestible energy concentration in growing pigs fed cereal grain-based diets', Journal Animal Science., 87, pp. 965-976.

Black, JL, Campbell, RG, Williams, IH, James, KJ \& Davies, GT 1986, 'Simulation of energy and amino acid utilization in the pig', Research and Development in Agriculture, vol. 3, pp. 121-145.

Cámara Mercantil de Productos del País. Montevideo, Uruguay CMPP. http://www.camaramercantil.com.uy/softis/documentos/dl/ alimbala/

Chen, L, Zhang HF, Gao, LX, Zhao, F, Lu, QP \& Sa, RN 2015, 'Effect of graded levels of fiber from alfalfa meal on intestinal nutrient and energy flow, and hindgut fermentation in growing pigs. Journal Animal Science, vol. 91, pp. 4757-4764.

De Blas, G, Gasa, J \& Mateos, GG 2013, Necesidades nutricionales para ganado porcino. Normas FEDNA, (segunda2 ${ }^{a}$ edición), Fundación Española para el Desarrollo de la Nutrición animal. http://www.fundacionfedna.org/sites/default/files/Normas $\% 20$ PORCINO_2013rev2.pdf

Ellis, M \& Augspurger, N 2001, 'Feed intake in growing-finishing pigs', Swine Nutrition, A. J. Lewis and L. L. Southern ed. CRC Press, Boca Raton, FL. Edited by Austin j. Lewis and L. Lee Sothern. second edition, pp. 447-467 ISBN 0-8493-0696-5

Edwards, SA 2003. 'Intake of nutrients from pasture by pigs', Proceedings of the Nutrition Society. Cambridge University Press, vol. 62, no. 02 , pp. $257-265$.

Edwards, SA 2005, 'Product quality attributes associated with outdoor pig production', Livestock Production Science, vol. 94, no. 1, pp. 5-14.

Fleming SE \& Lee, B 1983, 'Growth Performance and Intestinal Transit Time of Rats Fed Purified and Natural Dietary Fibers', Journal of Nutrition, vol. 113, pp. 592-601.
Folch, J, Lees, M \& Stanley, GHS. 1957, 'A Simple Method for the Isolation and Purification of Total Lipids from Animal Tissues'. Journal of Biological.

Forbes, J 2009, 'Integration of pre-and post-absorptive factors in feed intake regulation and prediction with particular respect to the pig', Voluntary feed intake in pigs, Chapter 3, Edited by: David Torrallardona and Eugeni Roura. Wagening Academic Publishers. The Netherlands, 2009. 365 p. ISSN 978-90-8686-096-8

Gaines, AM, Peterson, BA \& Mendoza, OF 2012, Herd management factors that influence whole herd feed efficiency, In: Feed efficiency in swine, edited by JF Patience Wageningen Academic Publisher, The Netherland, pp. 15-36

Gong, J \& Yang, C 2012, 'Advances in the methods for studying gut microbiota and their relevance to the research of dietary fiber functions', Food Research International, vol. 48, no.2, pp. 916-929.

Gregory, PC 2002, 'Role of the intestine in regulation of food intake in growing pigs', Chapter 14, Biology of the Intestine in Growing Animals, pp. 427-464.

Guillon, F \& Champ, M, 2000, 'Structural and physical properties of dietary fibres, and consequences of processing on human physiology', Food Research International, vol. 33, no.3, pp. 233-245.

Guillon, F, Saulnier, L, Robert, P, Thibault, JF \& Champ, M 2007, Chemical structure and function of cell wall through cereal grains and vegetable samples. In: Dietary Fibre Components and Functions, Edted by:Salovaara H, Gates F, Tenkanen M, Wageningen Academic Publishers, Wageningen, The Netherlands. pp. 31-64

Hansen, LL, Claudi-Magnussen C, Jensen SK, Andersenet HJ, 2006, 'Effect of organic pig production systems on performance and meat quality', Meat Science, vol.74, no. 4, pp. 605-615.

Ivarsson, E, Frankow-Lindberg, BE, Andersson, HK \& Lindberg, JE 2010a, 'Effect of inclusion level of chicory (Cichorium intybus $\mathrm{L}$ ) and ribwort (Plantago lanceolata L) forage in a cereal-based diet on dietary fibre digestibility in weaned piglets of different age', Livestock Science, vol. 134, no. 1-3, pp. 202-204.

Ivarsson, E, Frankow-Lindberg, BE, Andersson, HK \& Lindberg, JE 2010b, 'Growth performance, digestibility and fecal coliform bacteria in weaned piglets fed a cereal-based diet including either chicory (Cichorium intybus L) or ribwort (Plantago lanceolata L) forage' Animal, vol. 5, no. 04 , pp. 558-564.

Le Goff, G, Dubois, S, van Milguen, J \& Noblet, J 2002, 'Influence of dietary fibre level on digestive and metabolic utilization of energy in growing and finishing pigs', Animal Research, vol. 51, no. 3, pp. 245-259.

Lindberg, JE 2014, 'Fiber effects in nutrition and gut health in pigs, Journal of Animal Science and Biotechnology'. Journal of Animal Science and Biotechnology, vol. 5, no. 15 pp.1-7.

Lyu, ZQ, Huang, CF, Li, YK, Li, PL, Liu, H, Chen, YF, Li, DF \& Lai, CH 2018 , 'Adaptation duration for net energy determination of high fiber diets in growing pigs', Animal Feed Science and Technology, vol. 241 pp. 15-26.

Maichel, P \& Rérat, A 1998, 'Effect of adding sugar beet fibre and wheat bran to a starch diet on the absorption kinetics of glucose, aminonitrogen and volatile fatty acids in the pig', Reproduction Nutrition Development, vol. 38, pp. 49-68.

McDonald, DE, Pethick, DW, Mullan, BP \& Hampson, DJ 2001, 'Increasing viscosity of the intestinal contents alters small intestinal structure and intestinal growth, and stimulates proliferation of enterotoxigenic Escherichia coli in newly-weaned pigs', British Journal of Nutrition, vol. 86, pp. 487-498.

Moreno, LG \& Rosas, BSA 2012, 'Caracterización fenológica y nutricional de achicoria (Cichorium intybus) y llantén (Plantago lanceolata) para pastoreo', Tesis de grado, Universidad de la República, Facultad de Agronomía, Montevideo, Uruguay, vista 22 de agosto, 2018, http://164.73.52.13/iah/textostesis/2012/3820mor.pdf

Muniz, EME \& Silva, AD 2014, 'Inclusión de alfalfa en la dieta de ovinos en crecimiento alimentados con una ración totalmente mezclada: efecto en el comportamiento ingestivo y tasa de ingestión', Tesis de grado, 
Universidad de la República, Facultad de Veterinaria, Montevideo, Uruguay, vista 22 de agosto, 2018, http://www.fvet.edu.uy/images/ ContenidoMenu/Biblioteca/TesisdeGrado2014/FV-30915_0.pdf

Nilzén, V, Babol, J, Dutta, PC, Lundeheim, N, Enfält, A-C \& Lundström, $\mathrm{K} 2001$, 'Free range rearing of pigs with access to pasture grazing effect on fatty acid composition and lipid oxidation products', Meat Science, vol. 58, no.3, pp. 267-275.

NRC National Research Council 2012, Nutrient requirements of swine: Eleventh Revised Edition. Washington, DC: The National Academies Press. https://doi.org/10.17226/13298.

Noblet, J, Fortune, H, Shi, XS, \& Dubois, S 1994, Prediction of net energy value of feeds for growing pigs. J. Anim. Sci. 72:344-354.

Noblet, J \& Le Goff, G 2001, 'Effect of dietary fibre on the energy value of feeds for pigs', Animal Feed Science and Technology, vol. 90, no. 1, pp. 35-52.

Presto Åkerfeldt, M, Nihlstrand, J, Neil, M, Lundeheim, N, Andersson, HK \& Wallenbeck, A 2019, 'Chicory and red clover silage in diets to finishing pigs -influence on performance, time budgets and social interactions', Organic Agriculture, 9(1), pp. 127-138. doi: 10.1007/ s13165-018-0216-z.

Sun, X, Andrew, IG, Joblin, KN, Harris, PJ, McDonald, A \& Hoskin SO 2006, 'Polysaccharide compositions of leaf cell walls of forage chicory (Cichorium intybus L.)', Plant Science, vol. 170, pp. 18-27.
Terevinto, A, Cabrera MC \& Saadoum A 2019, 'Oxidative stability, fatty acid composition and health lipid indices of Longissimus dorsi muscle from Aberdeen Angus steers produced in different feeding systems', Ciência Rural. Universidade Federal de Santa Maria, vol. 49, no. 12. doi: 10.1590/0103-8478cr20190537.

Van Soest, PJ, \& Wine RH 1967, Use of detergents in the analysis of fibrous feeds. IV. Determination of plant cell-wall constituents. J. Assoc. Off. Anal. Chem. 50:50-55.

Viana, S, dos Reis, LM, Schultz, C, Soares, MH, Teixeira, D, Amorim, G \& Saraiva, A 2019, 'Metabolizable energy levels in diets with high lysine for growing and finishing pigs', Seminar: Ciências Agrárias, no. 1, p. 365, viewed 12September 2019, <http://search.ebscohost.com. proxy. timbo.org. vy:2048/login.aspx?direct=true $\& d b=e d s d o j \& A N=e d s d o$ j.38082b3f64454d77ac27d3fecdb37c1 4\&lang=es\&site=eds-live>. Wüstholz, J, Carrasco, S, Bergera, U, Sundrumb, A, \& Bellofet, G 2017. 'Fattening and slaughtering performance of growing pigs consuming high levels of alfalfa silage (Medicago sativa) in organic pig production', Livestock Science, 200, pp. 46-52. doi: 10.1016/i. livsci.2017.04.004. 